\title{
Inferring the Presence of Freezing Drizzle Using Archived Data from the Automated Surface Observing System (ASOS)
}

\author{
Scott D. Landolt, ${ }^{a}$ Andrew Gaydos, ${ }^{a}$ Daniel Porter,${ }^{\mathrm{b}}$ Stephanie DiVito, ${ }^{\mathrm{c}}$ Darcy Jacobson, ${ }^{\mathrm{a}}$ \\ ANDREW J. SCHWARTZ, ${ }^{d}$ Gregory ThOMPSOn, ${ }^{a}$ AND JOSHUA LAVE ${ }^{\mathrm{a}}$

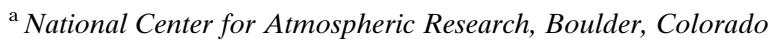 \\ ${ }^{\mathrm{b}}$ National Weather Service, Albuquerque, New Mexico \\ ${ }^{\mathrm{c}}$ William J. Hughes Technical Center, Federal Aviation Administration, Atlantic City, New Jersey \\ ${ }^{\mathrm{d}}$ University of Queensland, Brisbane, Queensland, Australia
}

(Manuscript received 24 June 2020, in final form 22 September 2020)

\begin{abstract}
In its current form, the Automated Surface Observing System (ASOS) provides automated precipitation type reports of rain, snow, and freezing rain. Unknown precipitation can also be reported when the system recognizes precipitation is occurring but cannot classify it. A new method has been developed that can reprocess the raw ASOS 1-minobservation (OMO) data to infer the presence of freezing drizzle. This freezing drizzle derivation algorithm (FDDA) was designed to identify past freezing drizzle events that could be used for aviation product development and evaluation (e.g., Doppler radar hydrometeor classification algorithms, and improved numerical modeling methods) and impact studies that utilize archived datasets [e.g., National Transportation Safety Board (NTSB) investigations of transportation accidents in which freezing drizzle may have played a role]. Ten years of archived OMO data (2005-14) from all ASOS sites across the conterminous United States were reprocessed using the FDDA. Aviation routine weather reports (METARs) from humanaugmented ASOS observations were used to evaluate and quantify the FDDA's ability to infer freezing drizzle conditions. Advantages and drawbacks to the method are discussed. This method is not intended to be used as a real-time situational awareness tool for detecting freezing drizzle conditions at the ASOS but rather to determine periods for which freezing drizzle may have impacted transportation, with an emphasis on aviation, and to highlight the need for improved observations from the ASOS.
\end{abstract}

KEYWORDS: Freezing precipitation; Algorithms; Automatic weather stations; Instrumentation/sensors; Measurements; Surface observations

\section{Introduction}

The Automated Surface Observing System (ASOS) is one of the primary weather observing systems in the United States, with over 900 stations across the country. These systems are maintained and supported by the National Weather Service (NWS), Federal Aviation Administration (FAA), and Department of Defense (DOD). Most of these systems are located at airports and provide meteorological observations used in aviation routine weather reports (METARs) that are critical for aircraft operations. The ASOS provides standard meteorological measurements of temperature, pressure, humidity, wind velocity, sky condition, visibility, obstructions to visibility, and liquid equivalent precipitation accumulation. In its current state, the ASOS can also provide a limited determination of pristine (i.e., non-mixed-phase) precipitation types-namely, rain, snow and freezing rain. Human observers at a limited number of the ASOS stations provide other reported precipitation types, such as drizzle, freezing drizzle, ice pellets, hail, and mixed-phase precipitation (FAA 2020).

In early 2015, the FAA issued a new certification rule regarding aircraft operations in supercooled large drop (SLD) icing conditions for a portion of new aircraft, including Part 25 aircraft less than $60000 \mathrm{lb}(1 \mathrm{lb}=\sim 0.45 \mathrm{~kg})$ or with reversible flight controls (FAA 2016). This new rule places limitations on

Corresponding author: Scott Landolt, landolt@ucar.edu aircraft operations in freezing-precipitation conditions (i.e., SLD, to include freezing drizzle and freezing rain) that were not previously included in the existing aircraft certification (Jeck 2002), impacting operations in icing conditions. These limitations to aircraft operations apply to takeoff, ascent, en route, descent, and landing (FAA 2016). This has created a present weather reporting shortfall at airports without human observers because the ASOS does not have the ability to directly detect and report drizzle, freezing drizzle, or ice pellets. As a result, pilots may not have the information necessary to avoid icing conditions for which they are not certified and may inadvertently fly into such conditions, exposing them to a safety risk.

To quantify the shortfall in freezing drizzle observations, and to develop a database of freezing drizzle events where aviation and surface transportation may have been impacted, a study was undertaken to develop a technique that could infer the presence of freezing drizzle conditions utilizing a combination of the various sensors available as part of the ASOS instrument suite. The reports of freezing drizzle can be used to attempt to quantify how frequently these precipitation types may occur, particularly at sites without human observers, underscoring the need for an ASOS sensor that can accurately detect freezing drizzle conditions.

\section{a. Freezing drizzle impacts on transportation}

Rasmussen et al. (2006) identified freezing drizzle as an icing hazard to aircraft on the ground that can cause ice accretion on 
jet engine fan blades and spinners. This can potentially lead to engine damage as the ice is shed during taxi and takeoff. In flight, ice can accrete on aircraft surfaces, thus decreasing lift and increasing drag as well as significantly affecting the airfoil shape and aerodynamics, leading to an increased risk of an accident. Flight through freezing drizzle can be especially hazardous (Shin and Berkowitz 1994; Tran et al. 1995). One such example was the 1994 ATR72 fatal crash near Roselawn, Indiana, that occurred after the aircraft was placed in a holding pattern in freezing drizzle conditions (Marwitz et al. 1997). A review of the National Transportation Safety Board (NTSB) aviation accident database revealed at least 28 aircraft accidents with 35 fatalities have occurred since 2000 in which freezing drizzle played a role or was the direct cause of the accident (NTSB 2020).

Freezing drizzle can also have significant impacts on surface transportation. The U.S. Department of Transportation's Federal Highway Administration (FHWA) provides information on weather-related accident statistics, including conditions created by freezing drizzle. Between 2007 and 2016, over 500 people were killed and over 156000 accidents were reported on icy pavement caused by freezing rain, freezing drizzle, and other contributing atmospheric conditions (e.g., wet roads that later froze) (Federal Highway Administration 2020). Malin et al. (2019) discussed the probability of various types of precipitation occurring and their relative automobile accident risk. Their analysis showed that freezing drizzle has a relatively low probability of occurrence but is a high-risk factor to surface transportation when it occurs. Tobin et al. (2019) compared vehicle related fatalities with weather conditions and noted problems when comparing the output from ASOS with traffic accidents because of ASOS limitations with precipitation types other than snow and rain.

These studies all highlight the impacts that freezing drizzle can have for both surface and aviation transportation. They further identify the need for accurate, timely reports of freezing drizzle conditions, their frequency of occurrence, and the need for determining whether freezing drizzle played a role in weather-related accidents.

\section{b. Current ASOS reporting capabilities}

To determine which sensors could be useful in inferring the presence of freezing drizzle, it is important to first understand how the ASOS currently determines occurrences of rain, snow and freezing rain. Rain and snow observations from the ASOS are derived using a combination of data from the Light Emitting Diode Weather Identifier (LEDWI) sensor (Fig. 1) and ambient air temperature (to further discriminate between rain and snow). The LEDWI operates by analyzing the frequency change in an emitted light beam. Hydrometeors that pass through the beam introduce different frequencies into the beam that are a function of the size and fall velocity of the hydrometeors (Starr and Wang 1989; Wade 2003). The LEDWI measures these frequency changes and determines the type of precipitation based on prior established relationships between frequency changes in the light beam and precipitation type. It is important to note that the LEDWI does not have the ability to directly measure or output the actual particle size.

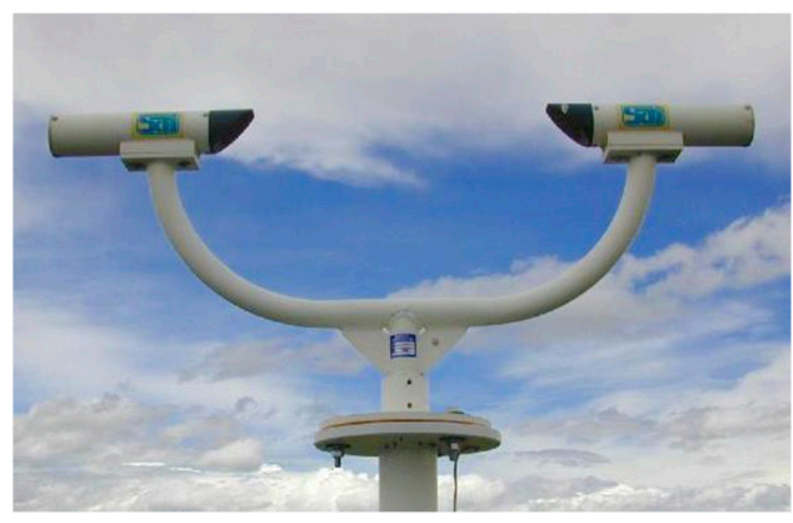

FIG. 1. LEDWI sensor used on the ASOS.

Because of this, it is common for the LEDWI to report "unknown precipitation" when the measured frequency change in the light beam does not match any of the expected relationships (NOAA 1998). Some of the common conditions associated with unknown precipitation reports from the LEDWI include mixedphase precipitation (e.g., rain combined with snow), precipitation phase transitions from one type to another, or light intensity precipitation that is near the limits of detection (approximately $1 \mathrm{~mm}$ in diameter) by the sensor.

In addition to reporting rain or snow, the ASOS can also identify freezing rain using a combination of several of the ASOS instruments. Freezing rain will be reported when the LEDWI reports rain or unknown precipitation, the ambient air temperature is less than $37^{\circ} \mathrm{F}\left(2.8^{\circ} \mathrm{C}\right)$ and the Goodrich Sensor System's 872C3 Icing Sensor, hereinafter referred to as the icing sensor (Fig. 2), detects active ice accretion (NOAA 1998). The icing sensor uses a cylindrical probe, also known as a magnetostrictive oscillator, that is electrically stimulated to vibrate at its resonant frequency near $40 \mathrm{kHz}$. The probe is oriented vertically to provide optimal exposure to freezing precipitation from any direction. When ice freezes on the cylindrical probe, the additional mass of the accreted ice causes a decrease in the resonant frequency (Fig. 3). This frequency decrease is directly proportional to the amount of ice that has accumulated on the cylindrical probe (Tattelman 1980, 1982). The optimal measurement range of the sensor is $40-39.4 \mathrm{kHz}$. Once the frequency approaches the low end of the detection range, the sensor initiates a de-icing cycle that heats the probe to remove the ice, returning the sensor back to its nominal frequency near $40 \mathrm{kHz}$. The sensor must cool back to the ambient temperature before it can begin accreting ice again, a process that can take up to $30 \mathrm{~min}$ in some cases (although $10 \mathrm{~min}$ or less is more common) and is one of the recognized drawbacks to using this type of technology. During the deicing cycle, accretion rates cannot be calculated. An extended cooldown time of the sensor could result in an automated algorithm mistakenly reporting a premature end to a freezingprecipitation event.

Once the ASOS has determined the type of precipitation occurring (rain, snow, freezing rain, or unknown precipitation), the observation is reported in widely distributed METARs. 


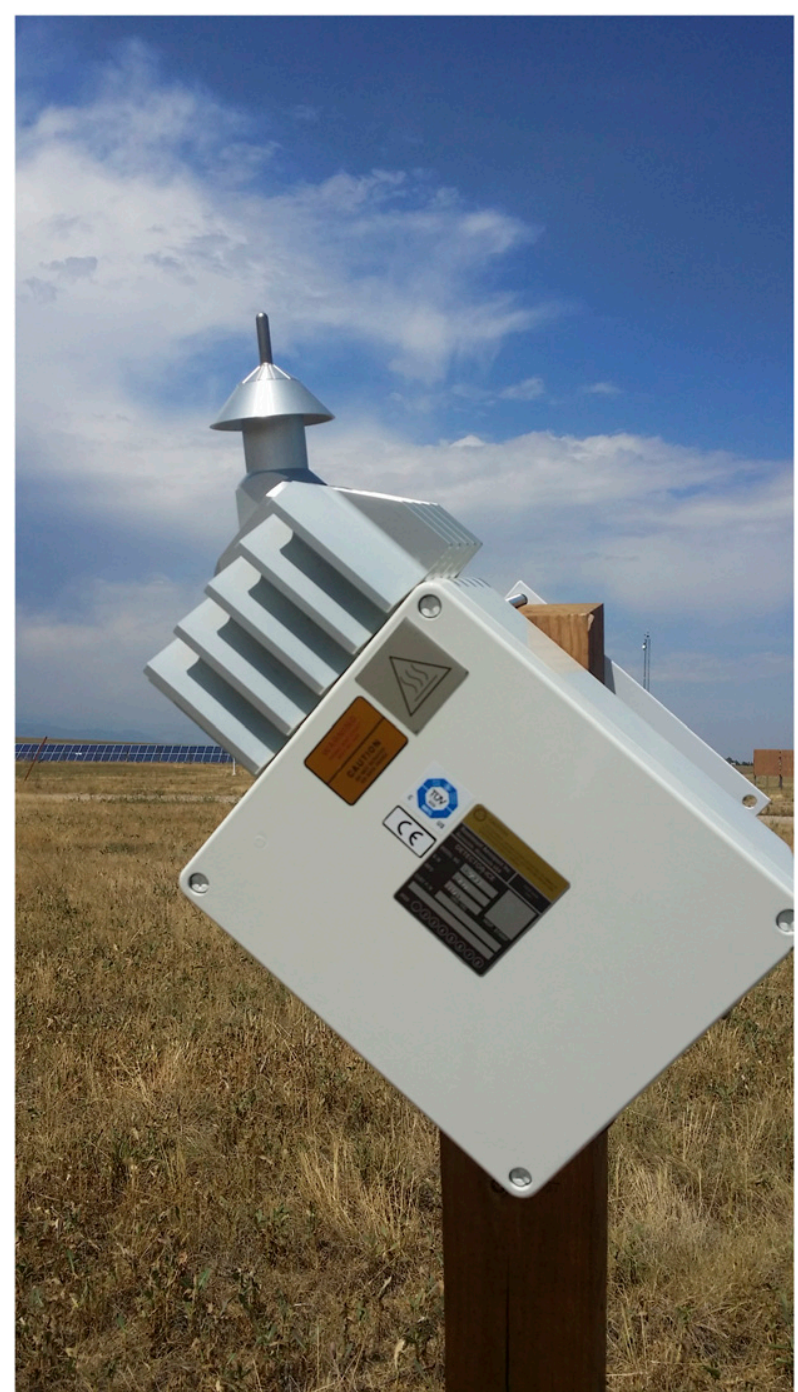

FIG. 2. Icing sensor used on the ASOS. The small, vertically pointing rod at the top is the actual probe that is used for detecting icing conditions.

However, METAR observations often contain precipitation types that the ASOS alone cannot report (e.g., freezing drizzle, ice pellets, and others including mixed-phase observations). These types of observations are reported by a human observer, also known as a contract weather observer (CWO), who is located at an airport with the ASOS and can augment and/or correct the ASOS present weather observation (e.g., when the ASOS reports unknown precipitation). CWOs are full-time weather observers that report weather conditions $24 \mathrm{~h}$ per day and 7 days per week at high-impact airports (i.e., high traffic airports, primarily serving commercial aircraft, where accurate weather observations are critical to operations). Airports with CWOs are classified as service level A or B airports (FAA 2020). Due to the funding expenses associated with human observers, approximately 130 ASOS sites across the country have CWOs. This accounts for only $12 \%$ of all the ASOS locations.

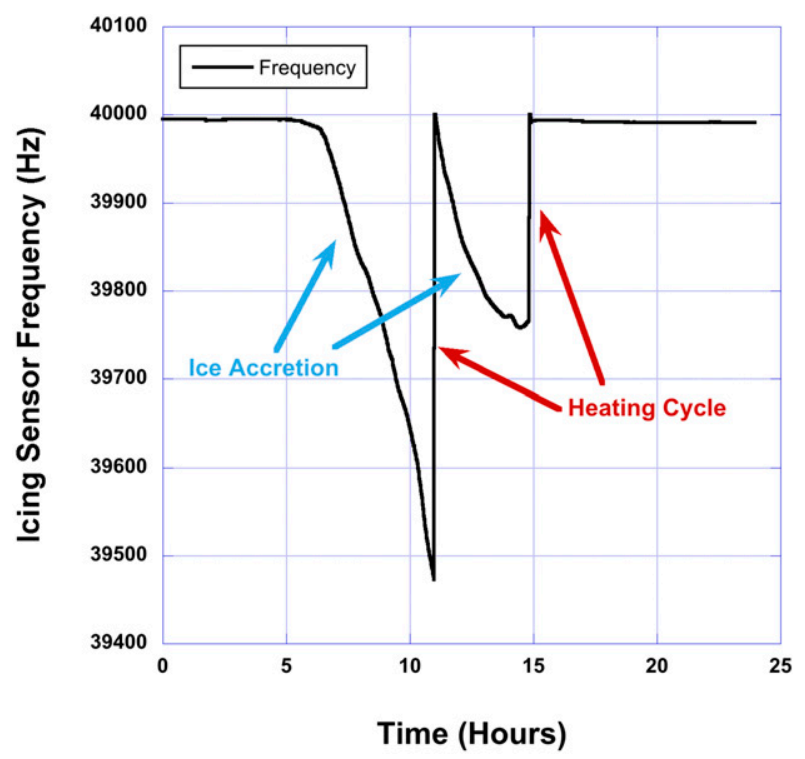

FIG. 3. Example plot of icing sensor frequency drops and heating cycles during a freezing-precipitation event.

Service level C airports account for another $34 \%$ of the ASOS locations and have observers that provide augmented observations on a limited basis. Observers at these airports are FAA Air Traffic Control (ATC) personnel trained to take weather observations. Typically, they provide these observations during the regular working day or during peak air traffic periods. However, these observers are prohibited from leaving the FAA ATC tower to observe the weather conditions and can only perform and provide weather observations as time permits during their regular duties.

Service level D airports compose the remaining $54 \%$ of the ASOS-equipped airports. These airports are generally small, have no ATC tower, and do not have human observers to report or augment weather conditions. Note that most, but not all, service level D ASOS sites are equipped with an icing sensor. As a result, weather reports at these sites are restricted to rain, snow and freezing rain (at sites with an icing sensor) as derived from the ASOS. Any precipitation detected by the ASOS beyond those types at service level D locations will be reported as unknown precipitation. If the precipitation is too light to be detected by the ASOS at these locations (common with drizzle, freezing drizzle or light snow), no precipitation type may be reported. This scenario poses a potential hazard for pilots if they do not know these conditions exist at an airport to or from which they are flying. This is especially true if their aircraft is not certified for flight in these conditions, or if they are not aware these precipitation types may exist but cannot be reported at these airports.

To quantify the importance of measuring freezing drizzle, data from METAR reporting stations across the United States over a 10-yr period (2005-14) were analyzed to determine the frequency of occurrence of the various pristine precipitation types (Fig. 4a). Not surprisingly, rain and snow account for the majority of precipitation type reports while freezing drizzle 
a)

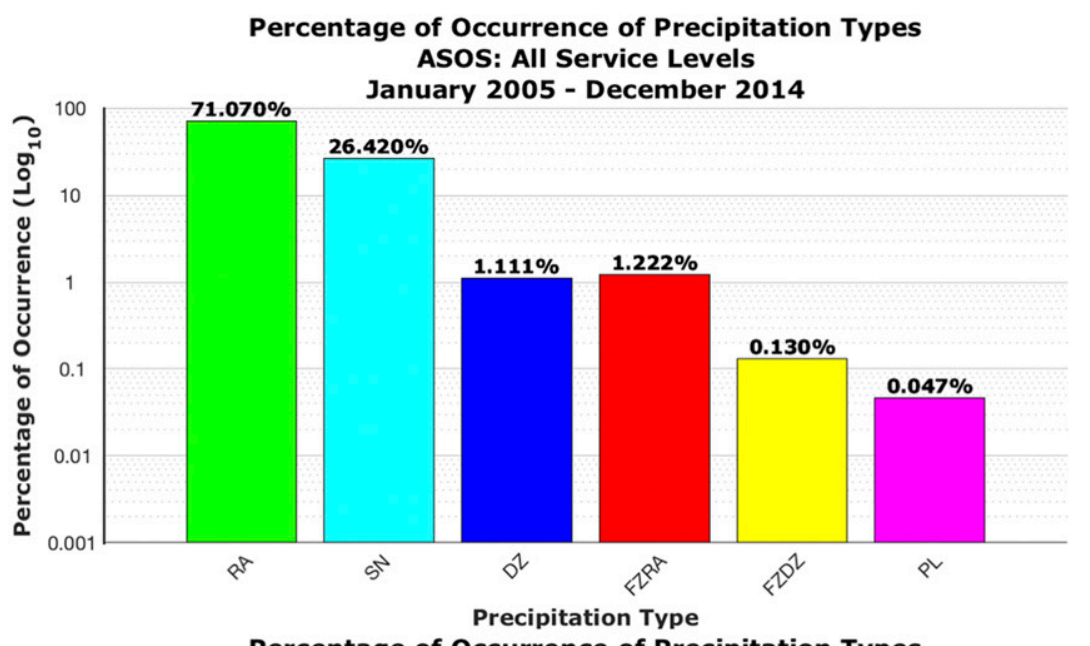

b)

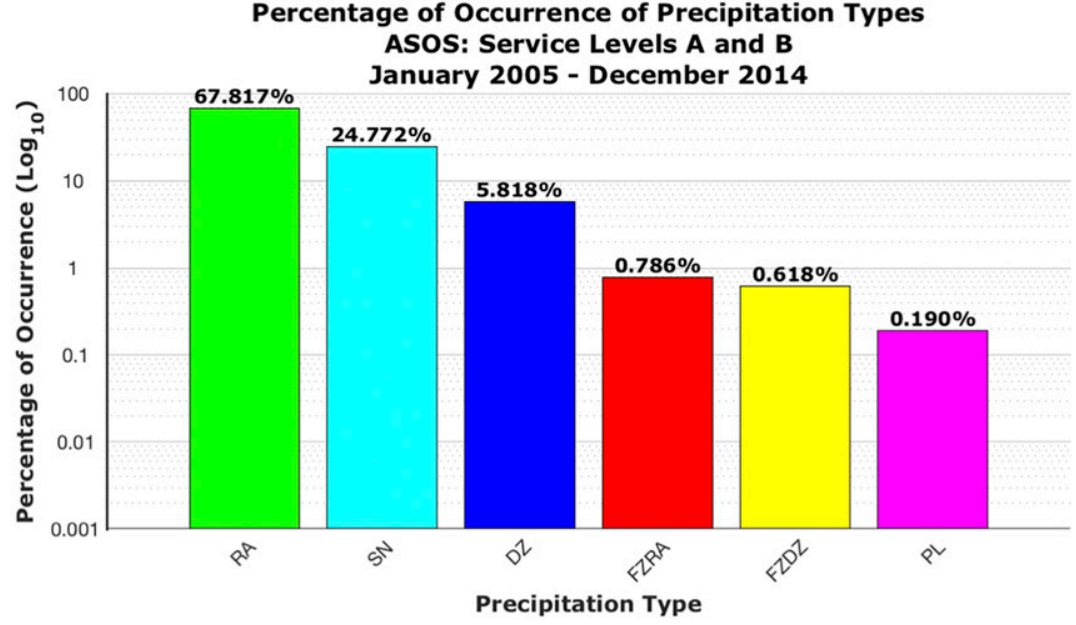

FIG. 4. (a) Percentage of occurrence of each precipitation type that was reported on the basis of METAR observations across the United States from 2005 to 2014 for all ASOS locations. Rain (RA), snow (SN), drizzle (DZ), freezing rain (FZRA), freezing drizzle (FZDZ), and ice pellets (PL) are the six precipitation types shown. Note that the $y$ axis is a logarithmic scale and was used to visualize the contributions of FZRA, FZDZ, and PL more easily. (b) Percentage of occurrence of each precipitation type that was reported on the basis of METAR observations from 2005 to 2014 for service level A and B airports only.

and ice pellets account for the least reported precipitation types. When examining these same percentages using METARs from service level A and B airports only, the percentages of drizzle, freezing drizzle and ice pellets all increase (Fig. 4b). This clearly indicates a shortfall in reporting these conditions at service level $\mathrm{C}$ and $\mathrm{D}$ airports and further indicates a low bias for these precipitation types due to the inability of the ASOS to report these conditions.

In another study, Landolt et al. (2019) showed a comparison of the various reported precipitation types before and after the installation of the ASOS. They noted a significant drop in the number of freezing drizzle reports after the introduction of the ASOS, highlighting how the ability to report this precipitation type has significantly decreased in recent years (because of the removal of human observers and the ASOS's inability to report freezing drizzle). The data shown in Fig. 4, in combination with the analysis shown by Landolt et al. (2019) further highlight the need for improved detection and reporting of freezing drizzle, especially at service level C and D airports. Additionally, the new FAA certification rule for aircraft operations in SLD icing conditions is expected to affect primarily smaller aircraft that often fly in and out of service level C and D airports. Therefore, as stated earlier, it becomes increasingly important to determine when freezing drizzle is occurring at all ASOS locations in an automated manner.

\section{c. Prior freezing drizzle algorithms for ASOS}

Since freezing precipitation will adhere to, and accrete on any surface with a subfreezing temperature, such as vegetation, pavement, and structures, a requirement for its detection is to determine if ice accretion is occurring. The icing sensor, through ice accretion measurements, has demonstrated the 
ability to detect surface icing conditions caused by freezing precipitation (rain and drizzle), freezing fog, wind-driven freezing mist, and hoarfrost (Ryerson and Claffey 1996; Ramsay 1997; SAIC 2001, 2003; Wade 2003). These studies have led to continued ASOS present weather algorithm research development to improve detection and reporting of various types of freezing precipitation.

The Raytheon Corporation developed a freezing drizzle algorithm for the ASOS in the late 1990s and early 2000s (Ramsay and Dover 2000; Ramsay 2002). This multisensor approach utilized different sensors on the ASOS, including the LEDWI and icing sensor as well as measurements of temperature, visibility and cloud cover, to infer the presence of freezing drizzle conditions. This algorithm underwent testing by the NWS but was not incorporated into the ASOS software.

The NWS and FAA also pursued a new present weather sensor replacement [referred to as the Enhanced Precipitation Indicator (EPI)] that was designed to detect drizzle, freezing drizzle, and ice pellet conditions. As part of the EPI activity, the NWS also pursued development of a freezing drizzle detection algorithm that would have incorporated the EPI sensor output with the icing sensor data to determine if freezing drizzle conditions were present. For example, if the EPI was reporting drizzle, the icing sensor was indicating accretion, and the temperature was sufficiently cold enough, freezing drizzle would be the reported observation. Unfortunately, testing of the EPI sensor determined it failed to meet the standards set by the FAA. As a result, the EPI was never added to the ASOS suite of sensors, and the algorithm was not implemented.

In another study conducted at the National Center for Atmospheric Research (NCAR), an algorithm was developed to detect both drizzle and freezing drizzle by reprocessing the raw LEDWI data (Wade 2003). This study showed that drizzle produced a unique, measurable signal in the LEDWI and that modifications to the signal processing algorithm could allow the LEDWI to report drizzle. Combining the observations of drizzle from the LEDWI with the measurements from the icing sensor would also allow for detection of freezing drizzle. Similar to the Raytheon algorithm, this algorithm also was not incorporated into the ASOS.

\section{d. Mist versus drizzle}

It is important to discuss briefly the difference between mist and drizzle because the two are often confused. This is especially the case in the aviation industry where pilots question why aircraft de/anti-icing fluids are not tested during "freezing mist" conditions. Mist, as used in METARs, is an obscuration to visibility and is reported when the visibility is between 7 and 5/8 mi (i.e., between 11.3 and $1 \mathrm{~km}$ ), and the dewpoint depression, if available, is less than $4^{\circ} \mathrm{C}$ (NOAA 1998). The American Meteorological Society (AMS) Glossary defines mist as "a suspension in the air consisting of microscopic water droplets or wet hygroscopic particles, reducing the visibility at Earth's surface to not less than $1 \mathrm{~km}$ or $5 / 8 \mathrm{mi}$." Drizzle is defined in the AMS Glossary as "a type (or form) of precipitation consisting of water droplets less than $0.5 \mathrm{~mm}(0.02 \mathrm{in}$.) in diameter and larger than 100 nm" (American Meteorological Society 2016). The important distinction is that drizzle, a precipitation process, falls to Earth's surface while mist is a suspension of particles in the air and is not associated with a precipitation process. Freezing drizzle is simply drizzle that falls in liquid form but freezes upon impact to form a coating of glaze (American Meteorological Society 2016). In the case of wind-driven freezing mist, it is assumed these particle sizes are similar to drizzle drop sizes and thus any accretion from them would be comparable to accretion from freezing drizzle.

\section{FDDA}

\section{Algorithm description}

The Freezing Drizzle Derivation Algorithm (FDDA) is a newly developed algorithm that uses the archived 1-minobservation (OMO) data from the ASOS to infer the presence of freezing drizzle. It is depicted in flowchart form in Fig. 5. If any condition in the tree is not met, the reported precipitation type will default to the ASOS output. The FDDA is loosely based on the algorithm described in Ramsay and Dover (2000), but utilizes additional instruments on the ASOS and has significantly more consistency checks to improve confidence in diagnosing freezing drizzle. It is not designed to operate in realtime situations due to the restrictions of accessing certain sensor data, discussed later. Additionally, because it only infers the presence of freezing drizzle, a better long-term solution to address the shortfall in operational observations would be to replace the present weather sensor on ASOS with a sensor that can directly detect freezing drizzle. The FDDA is designed to output only a single precipitation type, similar to the current ASOS present weather algorithm. It cannot report instances of mixed-phase conditions because it is using measurements from sensors that are themselves unable to distinguish between, or provide evidence of, multiple precipitation types simultaneously occurring. Also, because the algorithm relies on output from the icing sensor, the FDDA can only be used at ASOS locations that have this sensor.

Since the primary focus of the FDDA is to infer the presence of freezing drizzle, the FDDA first examines the output from the icing sensor to determine if any ice accretion is occurring. If there is no ice accretion, it is assumed nothing is adhering to the sensor and thus, no freezing precipitation (e.g., freezing drizzle or freezing rain) is occurring. However, if accretion is observed by the icing sensor, the FDDA then checks to determine if snow is being reported by the LEDWI. Wet snow can often adhere to the icing sensor probe, causing the frequency of the icing sensor to decrease. This can lead to a false accretion signal (NOAA 1998; Raytheon 1999; Ramsay and Dover 2000). Because of the priority the ASOS places on reporting snow, it currently disregards any reports of ice accretion from the icing sensor when the LEDWI reports snow. The FDDA uses this same logic.

If the icing sensor indicates accretion and the LEDWI does not indicate snow, sky cover is then examined to ensure that sufficient cloud cover is in place for reporting precipitation. Because overcast skies are typically expected during precipitation events, the FDDA examines the sky conditions for overcast observations (reported by the ceilometer) before 


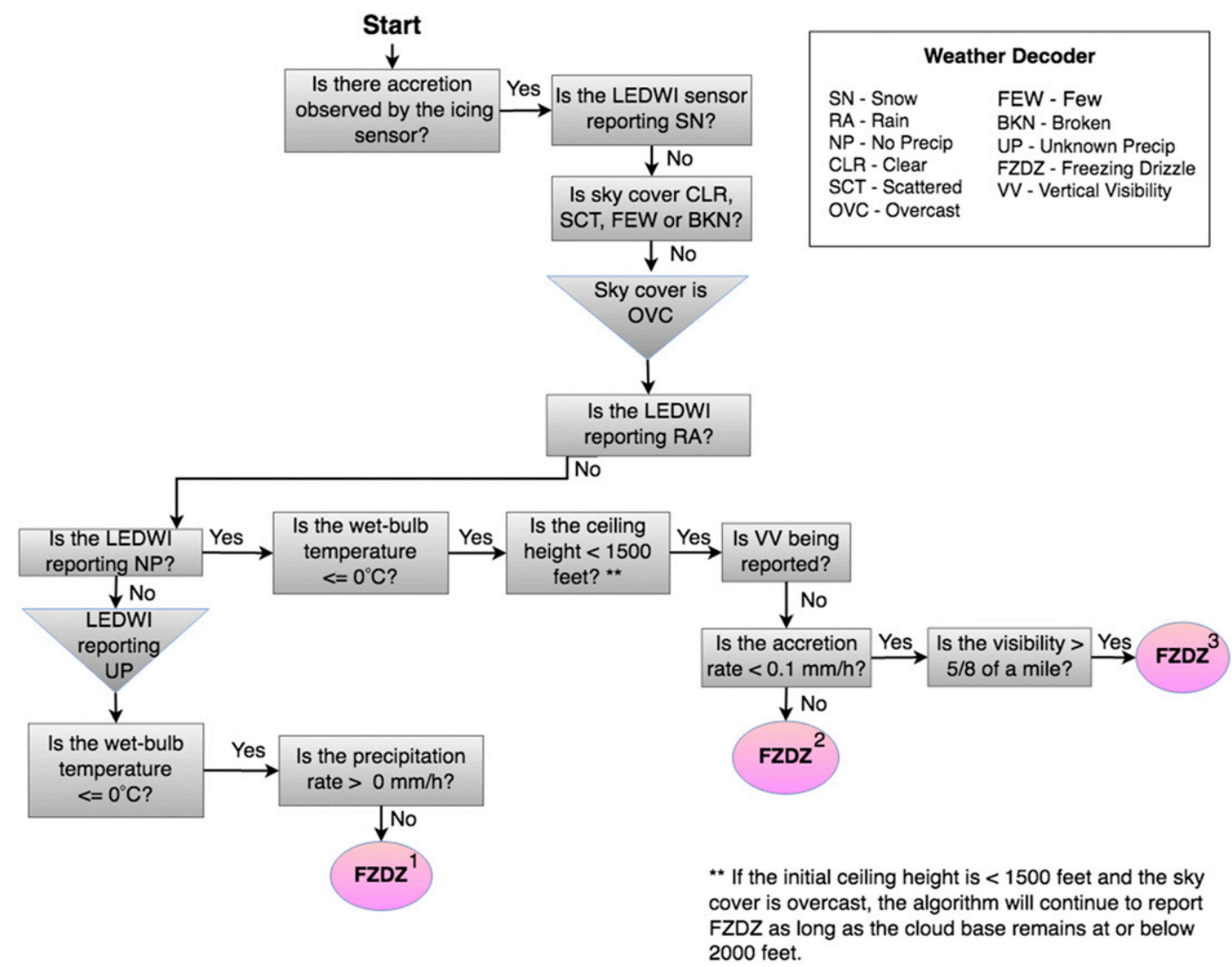

FIG. 5. Flowchart of the freezing drizzle derivation algorithm. The numbers to the upper right of each freezing drizzle precipitation identifier are shown to identify the logic path taken. For conditions not met, the algorithm would follow the normal ASOS logic for determining precipitation type.

continuing further to check the LEDWI observations. The methods for reporting sky conditions, including overcast, are explained in the ASOS user's manual (NOAA 1998). While it is possible to get freezing drizzle when the sky cover is not completely overcast, attempting to include those conditions in the algorithm led to significant increases in false detections, thus the algorithm was restricted to using only overcast skies.

After determining if the skies are overcast, the FDDA reexamines the output from the LEDWI. If the LEDWI reports rain, it is assumed that freezing rain is occurring because ice accretion was detected (the first decision point in the algorithm). If the LEDWI reports no precipitation (NP), but skies are overcast and ice accretion is detected by the icing sensor, freezing drizzle may be occurring. As stated earlier, the LEDWI cannot detect drizzle or freezing drizzle because drizzle-sized particles are typically just at or below the detection threshold of the sensor. Thus, the LEDWI sensor should report NP or unknown precipitation (UP) in these conditions. The FDDA uses this logic but includes additional checks to minimize false detections. If the LEDWI reports NP, the wetbulb temperature is calculated and ceiling heights and sky cover are examined. An analysis of ceiling heights was added after earlier versions of the FDDA indicated a significant number of false alarms for freezing drizzle accompanied by unrealistically high cloud bases. Most of these false reports were occurring in mountainous regions and were surmised likely to be due to frost formation in the valleys where high clouds could still be present.

To determine an appropriate ceiling height threshold for the FDDA, METAR reports of cloud-base height at service level $A$ and $B$ airports were analyzed when freezing drizzle was reported. Ceiling heights are not included in the OMO data; thus, ceiling heights were added using METAR data. Using data from the METARs often means only hourly reports of ceiling heights. However, changes in ceiling heights, particularly with lower cloud bases, will trigger a METAR SPECI. A SPECI is a METAR report issued when there is significant deterioration or improvement in airport weather conditions (FMH-1 2019). This includes changes in cloud-base height. Because the SPECI will report at nonroutine times compared to standard METARs, their inclusion should capture the changing conditions sufficiently to minimize the errors in the analysis. Figure 6 shows a cumulative frequency plot of the reported ceiling heights during freezing drizzle events from 2005 to 2014. Because greater than $90 \%$ of the reports occurred when cloudbase heights were $\leq 1500 \mathrm{ft}(\sim 460 \mathrm{~m})$, this threshold was incorporated into the FDDA as a requirement for reporting freezing drizzle. Additional analysis of the data indicated that cloud bases often rose near the end of the freezing drizzle event. To prevent the FDDA from prematurely ending reports 


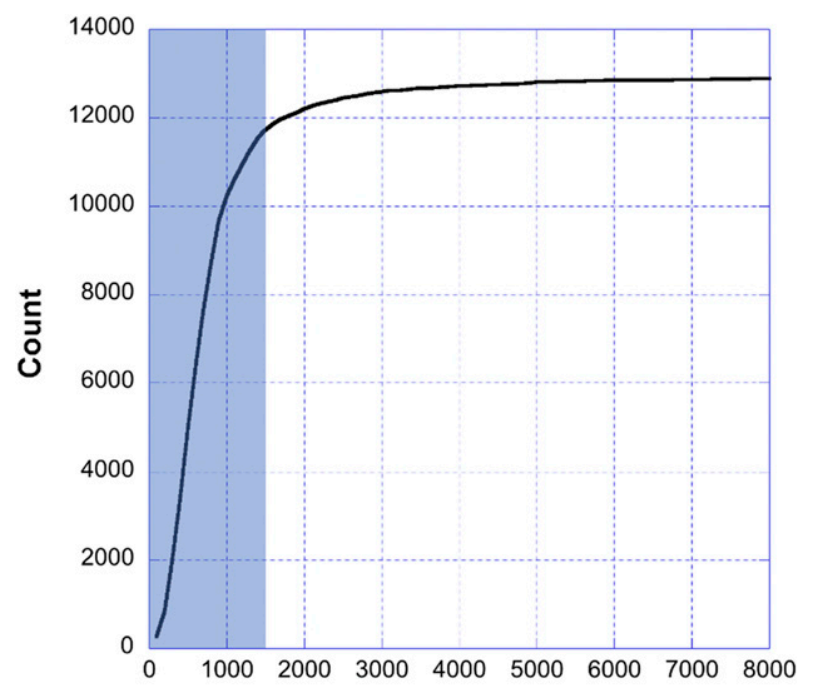

\section{Cloud Base Heights (ft)}

FIG. 6. Cumulative frequency of 11 years of cloud-base heights during FZDZ events from service level A and B airports. The shaded area indicates the region that encompasses $90 \%$ of the data points.

of freezing drizzle, cloud bases were allowed to rise as high as $2000 \mathrm{ft}(\sim 610 \mathrm{~m})$ after the start of the freezing drizzle event before the FDDA would stop characterizing the condition as freezing drizzle. Checks for reports of vertical visibility (VV), which is typically used in METARs when the cloud-base height is obscured due to fog or heavy precipitation, were added primarily to prevent instances of freezing fog being misdiagnosed as freezing drizzle.

The final check in the FDDA is related to LEDWI reports of UP since all other LEDWI output options have been considered. As explained earlier, a LEDWI report of UP can indicate precipitation near the detection threshold of the sensor or mixed-phase precipitation. It is possible that a report of UP could occur during very light snow, but it is unlikely that very light snow conditions would cause a large enough decrease in the icing sensor reading to indicate an icing event. Therefore, the FDDA assumes that if the LEDWI reports UP, the cloud cover is overcast, and the icing sensor indicates active ice accretion, an icing event is occurring. If the calculated wet-bulb temperature is below $0^{\circ} \mathrm{C}$, the precipitation gauge output is examined to determine if any precipitation rates are being recorded. The FDDA assumes that drizzle rates would typically remain below the detection threshold of the gauge [0.01 in. (i.e., $0.254 \mathrm{~mm}$ ) $\mathrm{h}^{-1}$ ]. However, prior studies (e.g., Raytheon 1999; Rasmussen et al. 2006) have shown that heavy freezing drizzle rates can overlap with light freezing rain rates (although in this case, the LEDWI should be reporting RA, and not UP). Additionally, higher precipitation rates can be an indication of mixed-phase precipitation, which would also cause the LEDWI to report UP. Thus, to eliminate the potential for miscategorizing the precipitation type, freezing drizzle observations were restricted to instances where the precipitation gauge did not detect a discernable rate. Drawbacks to using a precipitation rate of zero are also discussed in section $4 \mathrm{~b}$.

\section{Results}

\section{a. Algorithm verification}

As shown in the FDDA flowchart in Fig. 5, and described in the previous section, there are three possible ways that freezing drizzle can be inferred. After processing the OMO data through the FDDA from every ASOS location from 2005 to 2014, the number of minutes of diagnosed freezing drizzle were analyzed to determine how frequently the FDDA routed through path 1 (LEDWI reported UP), path 2 (LEDWI reported NP and the accretion rate was $>0.254 \mathrm{~mm} \mathrm{~h}^{-1}$ ) and path 3 (LEDWI reported NP, the accretion rate was $<0.254 \mathrm{~mm} \mathrm{~h}^{-1}$ and the visibility was $>5 / 8 \mathrm{mi}$, or $1 \mathrm{~km}$ ). Approximately $44 \%$ of the time, freezing drizzle diagnoses occurred when the LEDWI reported UP (path 1). Freezing drizzle was diagnosed when the LEDWI reported NP $56 \%$ of the time (combination of paths 2 and 3). This indicates that more than half the time when freezing drizzle is occurring, it is below the detection threshold of the LEDWI.

To evaluate the performance of the FDDA, METAR reports of freezing drizzle were used on a minute-by-minute basis at service level A and B airports. Since the METARs do not report every minute, the minutes between recorded METARs were populated using the data from the last reported METAR. For example, if snow was reported at 0105 UTC, rain was reported at 0120 UTC and no precipitation was reported at 0145 UTC, the minute reports from 0105 to 0119 UTC would be snow, the minute reports from 0120 to 0144 UTC would be rain, and the minute reports starting at 0145 UTC would have no precipitation. This method was chosen because any pilot looking at a METAR would have to rely on the last reported precipitation type, even if the METAR is nearly $1 \mathrm{~h}$ old. Table 1 shows the breakdown of the METAR-reported precipitation types when the FDDA indicated freezing drizzle. The METAR reports of freezing drizzle agreed with the FDDA $29 \%$ of the time. This indicates the algorithm has some skill in detecting freezing drizzle conditions, but errors in the METARs (described in further detail later) are likely resulting in the comparison being lower than it otherwise might be.

While the METARs reported freezing drizzle only $29 \%$ of the time when the FDDA reported freezing drizzle, the reverse comparison was also evaluated. When the METAR observations indicated freezing drizzle, the FDDA agreed with the METARs nearly $47 \%$ of the time. This is further evidence that the FDDA has some skill in detecting freezing drizzle conditions.

\section{b. Freezing drizzle case study event: 15-16 Jan 2018}

A case study of a high-impact event in northeastern New Mexico, where both ground and aviation transportation were impacted, is presented to demonstrate the ability of the FDDA to correctly diagnose the presence of freezing drizzle. An arctic cold front moved across the eastern plains of New Mexico on 
TABLE 1. METAR reported precipitation types from service level $\mathrm{A}$ and $\mathrm{B}$ airports when the FDDA indicated freezing drizzle.

\begin{tabular}{lcc}
\hline \multicolumn{1}{c}{ Precipitation type } & METAR counts & Percentage \\
\hline Freezing drizzle & 153527 & $29.1 \%$ \\
Snow & 97110 & $18.4 \%$ \\
No precipitation & 160537 & $30.5 \%$ \\
Freezing fog & 72908 & $13.8 \%$ \\
Freezing rain & 31652 & $6.0 \%$ \\
Drizzle (nonfreezing) & 8389 & $1.6 \%$ \\
Rain (nonfreezing) & 3138 & $0.6 \%$ \\
Total & 527262 & $100 \%$ \\
\hline
\end{tabular}

15 January 2018 as the center of a strong 1050-hPa high pressure center surged southward toward the Canadian-U.S. border. As a result, subfreezing surface temperatures near $-6^{\circ} \mathrm{C}$ were observed in the wake of the front as it moved across northeastern New Mexico. An extensive stratus cloud deck developed in conjunction with upslope flow along the eastern slopes of the Sangre de Cristo Mountains and adjacent highlands, covering most of northeastern New Mexico.

Numerical weather model soundings from the 15 January 20181200 UTC Global Forecast System (GFS20), North American Model (NAM40), and High-Resolution Rapid Refresh (HRRR) numerical weather models for northeastern New Mexico indicated a saturated atmosphere below $700 \mathrm{hPa}$, with a relatively dry layer from 700 to $400 \mathrm{hPa}$ (Fig. 7a). Temperatures aloft within the moist layer (up to $700 \mathrm{hPa}$ ) across northeastern New Mexico, including Las Vegas Municipal Airport (KLVS), ranged from $-6^{\circ}$ to $-11^{\circ} \mathrm{C}$ during the late-morning hours, but lowered further due to ongoing cold-air advection. Satellite imagery indicated a widespread stratus cloud deck at low altitude as well as some cirrus clouds in this general region (Fig. 7b). Based on the topdown methodology (Huffman and Norman 1988; Ramer 1993), the most likely precipitation type expected across this region was freezing drizzle, potentially changing to snow as the probability for ice nucleation increased with decreasing temperatures. The cirrus clouds were mostly thin and very likely to be sufficiently separated from the lower-altitude saturated atmosphere. Thus, it was unlikely that ice crystals from the upper clouds could fall into the lower supercooled liquid water (SLW) cloud. This reduced the chances for riming that would have lowered the SLW content, allowing the freezing drizzle cloud to persist.

Figure 8 depicts the METAR observations from KLVS for 15-16 January 2018. This site is a service level D ASOS location and is equipped with an icing sensor. There are several things to note in the METAR observations. First, near the beginning of the period, there are several reports of UP highlighted in yellow. Beginning at 2249 UTC 15 January, mist (BR; highlighted in blue) is reported because of lowered visibilities, but no precipitation types are reported. A recent modification to the METARs allows the ASOS to report ice accretion rates when freezing rain is occurring, but also when snow or no precipitation is occurring (Ryerson and Ramsay 2007). This was done to make forecasters aware that icing conditions (and/or frost) may exist even though neither is reported from nonaugmented ASOS sites. Starting at 2306 UTC, ice accretion rates appear in the observations highlighted in pink (encoded), in addition to the observations of mist. The reports of mist and ice accretion continue until 0632 UTC 16 January, when snow is reported as the precipitation type.

Figure 9 shows the raw frequency and the derived ice accretion values from the icing sensor on the KLVS ASOS for the 15-16 January event. At approximately 2300 UTC 15 January,
NAM40 Forecast Sounding near Las Vegas, New Mexico 0300 UTC January 16, 2018

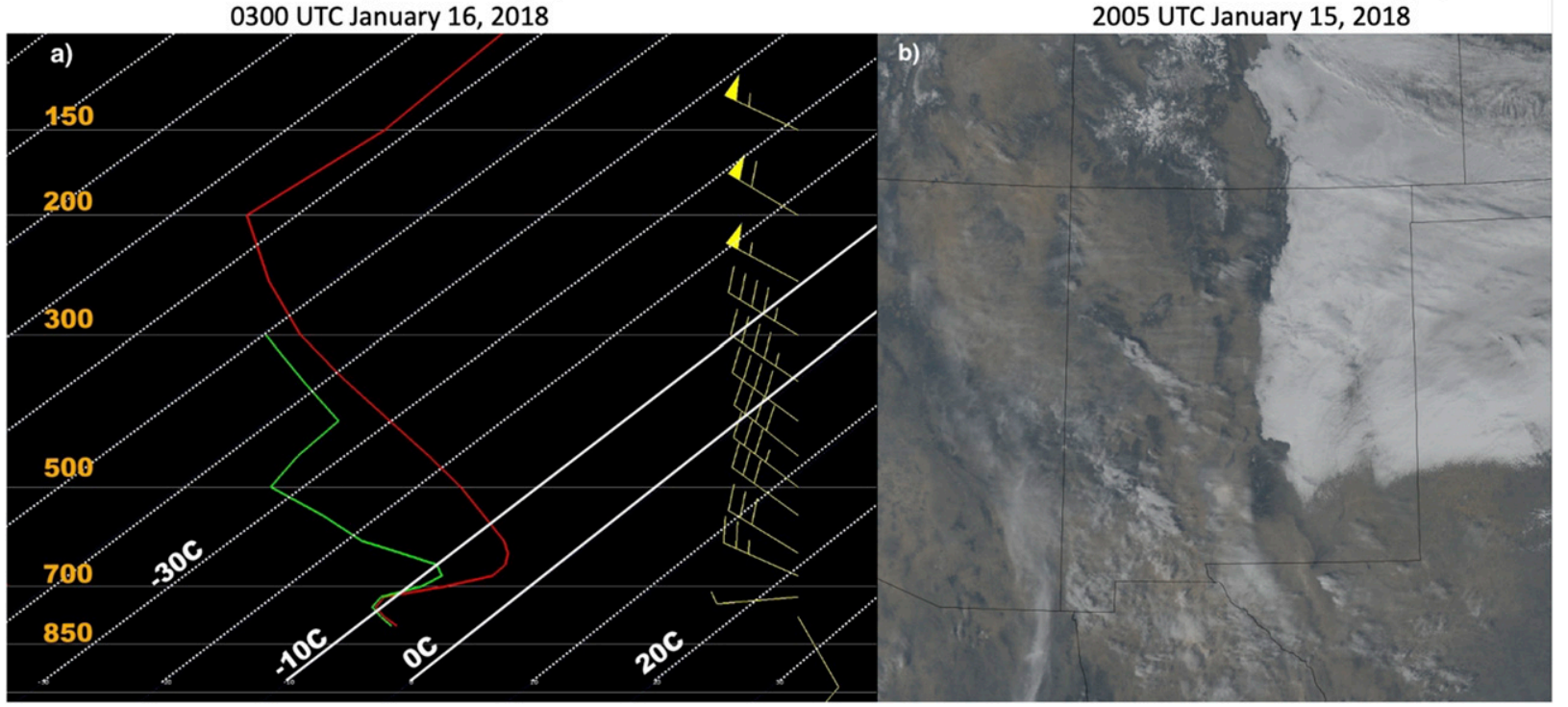

FIG. 7. (a) An example NAM40 1200 UTC forecast sounding during the freezing drizzle event for 0300 UTC 16 Jan near Las Vegas, and (b) GOES-16 satellite imagery showing a low-level stratus deck east of the mountains at 2005 UTC 15 Jan. 
KLVS 1522012 AUTO 14008KT 10SM OVC013 M03/M06 A3028 RMK AO2 T10331061

KLVS $152208 Z$ AUTO 14012KT 10SM UP SCT007 OVC013 M03/M06 A3028 RMK AO2 UPB04 P0000 T10331056

KLVS 152217Z AUTO 15012KT 10SM BKN005 OVC013 M04/M06 A3028 RMK AO2 UPB04E17 CIG 003 V009 P0000 T10391056

KLVS $152228 Z$ AUTO 13009KT 7SM UP BKN003 OVC007 M04/M06 A3029 RMK AO2 UPB04E17B25 CIG 002 V006 P0000

T10441056

KLVS $152249 Z$ AUTO 15009KT 2 1/2SM BR OVC002 M05/M06 A3030 RMK AO2 VIS 1 1/2V5 UPB04E17B25E35 P0000 KLVS $152253 Z$ AUTO 15010KT 2 1/2SM BR OVC002 M05/M06 A3030 RMK AO2 VIS 1 1/2V5 UPB04E17B25E35 SLP263 P0000 T10501056

KLVS $152306 Z$ AUTO 15012KT 1 1/2SM BR OVC002 M05/M06 A3030 RMK AO2 1000 T10501056

KLVS $152327 Z$ AUTO 15011KT 1/2SM FZFG OVC002 M06/M06 A3031 RMK AO2 |1001 T10561061

KLVS $152353 Z$ AUTO 15010KT 2 1/2SM BR OVC004 M06/M06 A3031 RMK AO2 SLP272 600001002 I6002 T10561061

110222105651010

KLVS $160014 Z$ AUTO 15010KT 3SM BR OVC006 M06/M07 A3032 RMK AO2 1001 T10611067

KLVS $160024 Z$ AUTO 15012KT 2 1/2SM BR OVC006 M06/M07 A3032 RMK AO2 |1002 T10611067

KLVS 160053Z AUTO 14010KT 2 1/2SM BR OVC005 M07/M07 A3035 RMK AO2 SLP285 1004 T10671072

KLVS $160153 Z$ AUTO 14009KT 2 1/2SM BR OVC006 M07/M08 A3037 RMK AO2 SLP303 1004 T10721083

KLVS $160253 Z$ AUTO 13009KT 3SM BR OVC008 M08/M09 A3040 RMK AO2 SLP324 I1003 |3011 T10781089 51025

KLVS $160323 Z$ AUTO 13007KT 2 1/2SM BR BKN007 OVC010 M08/M09 A3042 RMK AO2 [1001 T10831094

KLVS $160353 Z$ AUTO 12006KT 5SM BR BKN006 OVC018 M08/M09 A3042 RMK AO2 SLP331 l1002 T10831094

KLVS $160453 Z$ AUTO 11007KT 4SM BR OVC006 M09/M10 A3044 RMK AO2 SLP337 1001 T10891100 FZRANO \$

KLVS 160553Z AUTO 11006KT 7SM OVC006 M09/M10 A3044 RMK AO2 SLP336 [1001 I6015 T10891100 1105621089 $52004 \$$

KLVS $161110 Z$ AUTO 20006KT 9SM BKN004 OVC025 M09/M11 A3041 RMK AO2 1000 T10941106\$ KLVS $161119 Z$ AUTO 20007KT 8SM -SN SCT004 OVC025 M09/M11 A3041 RMK AO2 SNB12 P0000 1000 T10941106 \$ KLVS $161153 Z$ AUTO 19005KT 10SM OVC021 M09/M11 A3041 RMK AO2 SNB12E22 SLP337 P0000 60000 1000 I600 T10941111 110892109456004 \$

FIG. 8. METAR observations from KLVS for 15-16 Jan 2018. METAR observations between 0553 and 1110 UTC reported snow and are not shown here. Cyan coloring indicates periods for which mist (BR) was reported, yellow coloring indicates periods for which unknown precipitation (UP) was reported, magenta coloring indicates periods for which ice accretion was reported, and green coloring indicates where the freezing rain sensor became inoperative.

the frequency started to drop significantly, with ice accretion rates over $1 \mathrm{~mm} \mathrm{~h}^{-1}$ (Figs. 8 and 9). The frequency continued to decrease until approximately 1330 UTC 16 January, though the rate of decrease dropped significantly around 0800 UTC. As indicated in Figs. 8 and 9, ice accretion rates began at 2306 UTC, just after the start of the frequency decrease measured by the sensor. After 0553 UTC, the METARs indicate snow and the reports of icing cease. As discussed earlier, this is because of the noted problem with wet snow adhering to the sensor and causing false reports of accretion. In this particular case, with temperatures around $-10^{\circ} \mathrm{C}$ and the continued decrease in the icing sensor frequency, it is likely the actual conditions were a mixed-phase snow and freezing drizzle event.

As further evidence that freezing drizzle was likely occurring, around 0130 UTC 16 January, law enforcement officials closed both northbound and southbound lanes of Interstate 25 for several hours due to a fatal multivehicle collision that occurred as a result of icy roads roughly seven miles south of Las Vegas, New Mexico (Journal North Report 2018). At approximately 0330 UTC, an experimental single engine aircraft was en route from Liberal, Kansas, to Santa Fe, New Mexico, when it experienced severe icing at $9000 \mathrm{ft} \operatorname{MSL}(\sim 2.7 \mathrm{~km})$ and crashed approximately $8 \mathrm{mi}$. $(\sim 13 \mathrm{~km})$ southeast of Santa $\mathrm{Fe}$ (NTSB 2018). FAA inspectors noted the accumulation of structural ice on the airframe and ice along the debris path on the ground from the wreckage.

The archived OMO data from KLVS for this event were processed through the FDDA to determine if the algorithm would have flagged this event as freezing drizzle. Beginning at 2316 UTC and continuing through 0453 UTC, the FDDA reported freezing drizzle. At 0453 UTC, as indicated by the METAR observation of freezing rain sensor not operational (FZRANO) and noted in the plot in Fig. 8 in green, the icing sensor became inoperative for approximately $20 \mathrm{~min}$. When it started to report again, the frequency was over $40 \mathrm{kHz}$, and required extra time to drop below that threshold before the FDDA could once again report freezing drizzle.

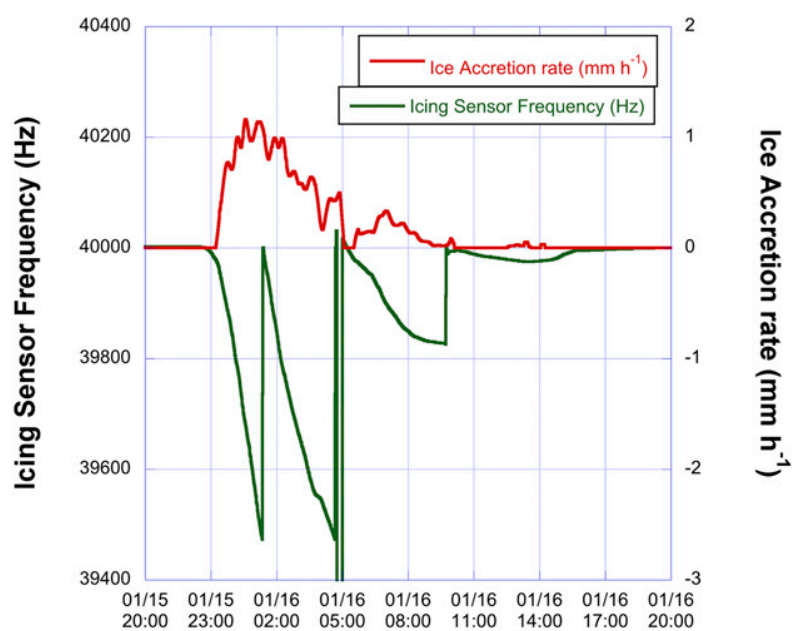

Time (UTC)

FIG. 9. Icing sensor frequency and derived ice accretion rates from KLVS for the 15-16 Jan 2018 event. Note that the sensor stopped working for approximately $0.5 \mathrm{~h}$ just before $0500 \mathrm{UTC}$ 16 Jan. 


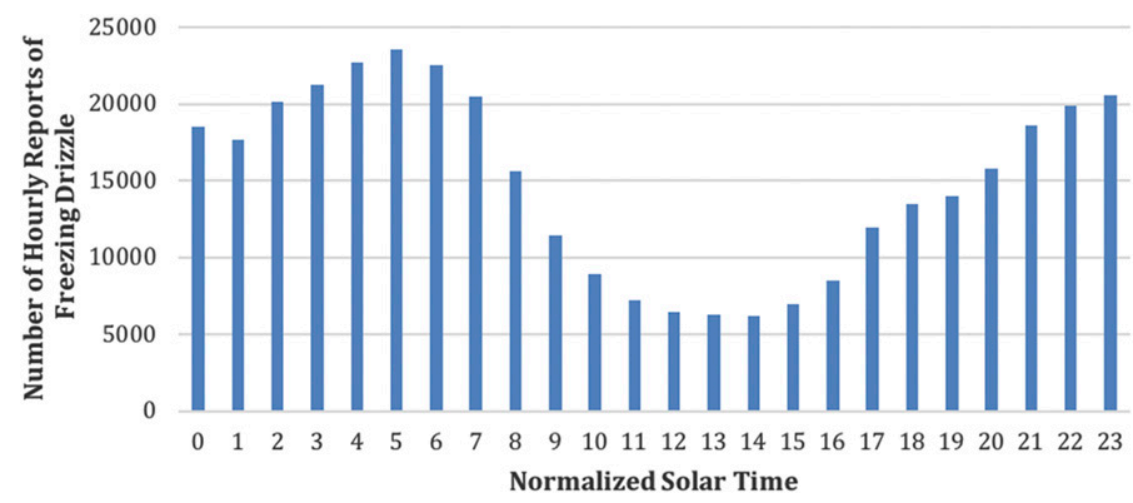

FIG. 10. Frequency of freezing drizzle occurrence by time of day in normalized solar time.

At approximately 0520 UTC, the FDDA again reports freezing drizzle until 0653 UTC, when the LEDWI reports snow. Had freezing drizzle been reported by the ASOS, it is possible preventative measures could have been taken in both transportation instances described above and safety hazards avoided.

\section{c. Freezing drizzle occurrence by time of day}

Prior research (e.g., Cortinas et al. 2004) found a peak in freezing drizzle occurrence near sunrise. The Cortinas study used a few METAR stations around the country and relied on the CWO observations of freezing drizzle in the METARs. No explanations were provided for the timing of the peak occurrence, but it is speculated that it could be related to CWOs first noticing the freezing drizzle as the sky begins to brighten in the morning. A similar analysis was undertaken utilizing the freezing drizzle reports from the FDDA to determine if this trend was also observed. Following the techniques used in the Cortinas study, all derived times of freezing drizzle were converted to normalized solar time (NST). NST converts standard times such that each station experiences an equal $12 \mathrm{~h}$ of light and $12 \mathrm{~h}$ of darkness. During the winter months, each daytime minute is stretched beyond a minute in NST time, and each nighttime minute is compressed to shorter than a minute such that an even $12 \mathrm{~h}$ of day and $12 \mathrm{~h}$ of night are achieved. For reference, 0600 NST is sunrise and 1800 NST is sunset. OMO data from 2005 to 2014 for all the ASOS stations across the country were processed with the FDDA. To eliminate spurious reports of freezing drizzle, only FDDA-derived events where freezing drizzle lasted $30 \mathrm{~min}$ or more were counted. Once the times for each station were converted to NST, multiple reports of freezing drizzle in any given hour were reduced to one so that hourly reports (not number of hours) of freezing drizzle could be analyzed. This was done to prevent observations such as SPECIs (METAR reports at times other than the standard reporting times) from artificially inflating the number of freezing drizzle reports.

Figure 10 shows a histogram depicting the number of hourly reports of freezing drizzle for each NST hour. Similar to the Cortinas study, the peak in freezing drizzle occurrence is near sunrise (at 0500 NST). These results further suggest the FDDA is correctly diagnosing freezing drizzle but also indicates the observed maximum in freezing drizzle at 0500 NST is a real phenomenon and is not a result of the CWOs detecting it as the sky brightens at dawn. The data were further broken down by regions (not shown) to determine if the cause was related to terrain. The peak was similar for mountainous versus flat regions, discounting terrain effects as a possible cause in the peak at $0500 \mathrm{NST}$. It is surmised this may be due to the diurnal temperature cycle, since the temperature is typically coldest near sunrise, but more research would be needed to confirm this hypothesis.

Interestingly, a secondary peak in freezing drizzle occurrence is shown near midnight. This was not observed in the Cortinas study and while the reasons for it are not obvious, one possible explanation is the algorithm is misclassifying other precipitation types as freezing drizzle (because of the previously stated issues with the LEDWI sensor).

\section{Discussion}

\section{a. Potential algorithm drawbacks}

Incorporating data from multiple sensors on the ASOS has minimized the potential for false indications of freezing drizzle, but errors still remain that could result in the algorithm misclassifying freezing drizzle. Relying on accurate measurements of precipitation can be problematic for the nearly 2/3 of ASOS sites that still use heated tipping-bucket gauges. These gauges can occasionally suffer from the buckets freezing up inside the gauge, resulting in no reported rates from the gauge. In this instance, it is possible that freezing rain could be misclassified as freezing drizzle if the LEDWI is not reporting RA, but rather UP. In the remainder of the $1 / 3$ of ASOS sites that use a weighing gauge, precipitation rate can still be determined as long as the orifice heater on the gauges is operational.

Another potential issue with the algorithm is that it assumes the output from the LEDWI sensor is correct. To determine if this was an issue, a separate analysis was performed on the LEDWI to determine how often the LEDWI agreed with the human observer at service level A and B airports for both snow and rain. This analysis presented a challenge because METAR observations do not indicate whether an observer changed any part of the METAR. Therefore, a separate algorithm was 
developed that is based on the information provided in the ASOS manual that mimicked how the ASOS uses the OMO data to determine the present weather type (NOAA 1998). METARs at service level A and B airports from 2005 to 2014 were then analyzed and periods of rain and snow were identified. The ASOS-derived METAR present weather reports were then time matched to the actual METAR present weather reports and compared. For snow, the ASOS-derived reports agreed with the METARs $67.7 \%$ of the time. For rain, the ASOS-derived reports agreed with the METARs $69.7 \%$ of the time. This indicates that the observers were augmenting the ASOS present weather reports approximately one-third of the time and suggests that reliance of the FDDA on the LEDWI reports can result in erroneous observations of freezing drizzle.

\section{b. METAR error sources}

Reports of NP accounted for over $30 \%$ of the METAR observations (Table 1). In these instances, it is possible that the freezing drizzle was too light for the observer to observe/report it (as documented in Rasmussen et al. 2006), or the observer identified it as an obscuration and reported it as such (e.g., mist). Obscurations, such as haze, mist and fog, were included in the NP category as long as no precipitation types were reported. Of these reported obscurations, mist accounted for $96.8 \%$, fog $2.1 \%$, and haze $1.1 \%$ of the NP METAR counts. It is interesting to note the very high percentage of mist reports. This clearly indicates that some atmospheric process (either precipitation or some other phenomena) is reducing the visibility and causing ice accretion, but the LEDWI is not sensitive enough to determine the cause of either. If the ice accretion (and reduction in visibility) is due to precipitation (e.g., freezing drizzle), then the observer is missing it or misclassifying it as an obscuration. Accounting for this error could replace some NP reports (where BR is reported) with freezing drizzle, increasing the freezing drizzle observations from the METARs. This would increase the agreement between the freezing drizzle algorithm and the METARs to more than just one-third of the time.

Interestingly, snow was reported in the METARs $18 \%$ of the time. If the LEDWI was reporting snow, the FDDA would have reported snow since snow overrides any observations of freezing precipitation in the FDDA (Fig. 5). Thus, these METAR reports were instances where the CWO overrode the ASOS-determined precipitation type with snow. This highlights the issue presented earlier regarding the drawback to the algorithm relying on the LEDWI to accurately report the precipitation type and provides further explanation why the agreement between the FDDA and the METARs is not higher.

Freezing fog reports also account for more than $10 \%$ of the METAR observations (Table 1). Similar to the previously described issues, these reports are likely a result of the CWOs changing the ASOS measured visibility reports to less than $5 / 8$ mile (changing the obscuration from mist to fog). The ASOS cannot detect freezing fog but will report freezing fog if visibility is less than $5 / 8$ mile $(1 \mathrm{~km})$ and the temperature is below freezing. While it is possible the observers are mischaracterizing freezing drizzle and freezing fog, this highlights the drawback to relying on visibility to make the distinction between freezing fog and freezing drizzle.

Another potential source of discrepancies could be that CWOs are not always collocated with the ASOS. At some airports, the CWOs may be on the opposite side of the airport (which could be more than a mile away) from the ASOS, leading to possible disparities between their observations and the ASOS observations.

\section{Conclusions}

Freezing drizzle and freezing rain are known hazards to both the surface and aviation transportation industries. While automated observations of freezing rain are possible, automated observations of freezing drizzle are not. This has created an observing shortfall that continues to result in property destruction and human casualties. A new method for inferring the presence of freezing drizzle using archived ASOS OMO data was presented. This method can be particularly useful in cases where freezing drizzle may have impacted surface transportation and aircraft operations. The FDDA has shown some skill in diagnosing freezing drizzle conditions when compared with METAR observations of freezing drizzle made by CWOs. Comparing the freezing drizzle observations from the FDDA to the same in METARs, and vice versa, shows an agreement of less than $50 \%$. However, some of these discrepancies are likely due to inherent errors from the ASOS sensors the FDDA must use to infer freezing drizzle (i.e., erroneous reports of precipitation type from the LEDWI). Discrepancies also exist in the METARs as CWOs can miss observing freezing drizzle or report a different environmental condition (such as an obscuration), leading to a lower agreement between the METARs and the FDDA than may actually exist.

Analysis of individual freezing drizzle cases (including the case study presented earlier), particularly high-impact events, has shown the FDDA can capture these events well. Additionally, the FDDA also showed good agreement with prior published research in reporting a peak of freezing drizzle prior to sunrise.

While the FDDA does show skill in detecting freezing drizzle events, to address properly the noted shortfalls, a new present weather sensor that can detect rain, snow and freezing rain in addition to freezing drizzle and other precipitation types is required to replace the LEDWI. Utilization of a new present weather sensor with the capabilities to detect these conditions, in combination with select parts of this new FDDA (particularly the checks to minimize false detections) would present a significant advancement in the ability of the ASOS to more accurately report the present weather conditions.

Acknowledgments. This research is in response to requirements and funding by the Federal Aviation Administration (FAA). The views expressed are those of the authors and do not necessarily represent the official policy or position of the FAA. NCAR is sponsored by the National Science Foundation. The authors acknowledge the support of James Riley of the FAA. We also acknowledge of the support of Karen Slater of NCAR for her editing efforts. 


\section{REFERENCES}

American Meteorological Society, 2016: Mist. Glossary of Meteorology, http://glossary.ametsoc.org/wiki/mist.

Cortinas, J. V., Jr., B. C. Bernstein, C. C. Robbins, and W. J. Strapp, 2004: An analysis of freezing rain, freezing drizzle, and ice pellets across the United States and Canada: 1976-90. Wea. Forecasting, 19, 377-390, https://doi.org/10.1175/15200434(2004)019<0377:AAOFRF > 2.0.CO;2.

FAA, 2016: Supercooled large drop icing conditions. U.S. Code of Federal Regulations, Title 14, Section 25.1420, https:// www.govinfo.gov/app/details/CFR-2016-title14-vol1/CFR-2016title14-vol1-sec25-1420.

__ 2020: Surface weather observing. FAA Order 7900.5E, http://www.faa.gov/regulations_policies/orders_notices.

Federal Highway Administration, 2020: How do weather events impact roads? Accessed 18 February 2019, https://ops.fhwa.dot.gov/ weather/q1_roadimpact.htm.

Huffman, G. J., and G. A. Norman Jr., 1988: The supercooled warm rain process and the specifications of freezing precipitation. Mon. Wea. Rev., 116, 2172-2182, https://doi.org/10.1175/15200493(1988)116<2172:TSWRPA > 2.0.CO;2.

Jeck, R. K., 2002: Icing design envelopes (14 CFR parts 25 and 29, appendix C) converted to a distance-based format. Federal Aviation Administration Office of Aviation Research Rep. AR-00/30, 55 pp.

Journal North Report, 2018: Two die in I-25 pileup involving 20 vehicles. Albuquerque Journal, 17 January, https:// www.abqjournal.com/1119978/two-die-in-chain-collision-oni-25-south-of-las-vegas-n-m.html.

Landolt, S. D., J. S. Lave, D. Jacobson, A. Gaydos, S. DiVito, and D. Porter, 2019: The impacts of automation on present weather-type observing capabilities across the conterminous United States. J. Appl. Meteor. Climatol., 58, 2699-2715, https://doi.org/10.1175/JAMC-D-19-0170.1.

Malin, F., I. Norros, and S. Innamaa, 2019: Accident risk of road and weather conditions on different road types. Accid. Anal. Prev., 122, 181-188, https://doi.org/10.1016/j.aap.2018.10.014.

Marwitz, J. D., M. K. Politovich, B. C. Bernstein, F. M. Ralph, P. J. Nieman, R. Ashenden, and J. Bresch, 1997: Meteorological conditions associated with the ATR72 aircraft accident near Roselawn, Indiana, on 31 October 1994. Bull. Amer. Meteor. Soc., 78, 41-52, https://doi.org/10.1175/1520-0477(1997) 078<0041:MCAWTA $>2.0 . \mathrm{CO} ; 2$.

NOAA, 1998: Automated Surface Observing System (ASOS) user's guide. National Weather Service Doc., 61 pp., http:// www.nws.noaa.gov/asos/pdfs/aum-toc.pdf.

NTSB, 2018: Crash during approach to landing, Bryk Steven L Velocity XL RG, N787SB, Santa Fe, New Mexico, January 15, 2018. Aircraft Accident Report NTSB/CEN18LA077, XX 40 pp., https://www.ntsb.gov/_layouts/NTSB/OpenDocument.aspx? Document_DataId=6903618\&FileName $=$ CEN18LA077_ weatherfactualSuffernfinal-Rel.pdf.

- 2020: Aviation accident database and synopses. Accessed 18 July 2018, https://www.ntsb.gov/_layouts/ntsb.aviation/ index.aspx.

Ramer, J., 1993: An empirical technique for diagnosing precipitation type from model output. Fifth Int. Conf. on Aviation Weather Systems, Vienna, VA, Amer. Meteor. Soc., 227-230.
Ramsay, A. C., 1997: Freezing rain detection and reporting by the Automated Surface Observing System (ASOS). Preprints, First Symp. on Integrated Observing Systems, Long Beach, CA, Amer. Meteor. Soc., J65-J69.

- 2002: Freezing drizzle (FZDZ) identification from the Automated Surface Observing System (ASOS): Status of the ASOS multi-sensor FZDZ algorithm. Sixth Symp. on Integrated Observing Systems, Orlando, FL, Amer. Meteor. Soc., 6.5, https://ams.confex.com/ams/annual2002/techprogram/ paper_27226.htm.

— , and J. Dover, 2000: Freezing drizzle identification from the Automated Surface Observing System (ASOS): Field evaluation of a proposed multi-sensor algorithm. Ninth Conf. on Aviation, Range, and Aerospace Meteorology, Orlando, FL, Amer. Meteor. Soc., 4.17, https://ams.confex.com/ams/ Sept2000/techprogram/paper_16133.htm.

Rasmussen, R. M., and Coauthors, 2006: New ground deicing hazard associated with freezing drizzle ingestion by jet engines. J. Aircr., 43, 1448-1457, https://doi.org/10.2514/1.20799.

Raytheon, 1999: Freezing drizzle algorithm development. Raytheon Rep., 21 pp.

Ryerson, C., and K. Claffey, 1996: Efficacy of ice detector hoarfrost observations. Proc. Fourth Annual Mt. Washington Observatory Symp., North Conway, NH, Mount Washington Observatory, 45-55.

— , and A. C. Ramsay, 2007: Quantitative ice accretion information from the Automated Surface Observing System. J. Appl. Meteor. Climatol., 46, 1423-1437, https://doi.org/ 10.1175/JAM2535.1.

SAIC, 2001: Quick-time report of ASOS icing event at Kansas City International (KMCI), February 23, 2001. SAIC Rep. W/OST32, 9 pp.

- 2003: Case study of icing event at Denver, Colorado (KDEN), October 31-November 1, 2002. SAIC Rep. W/OST32, 14 pp.

Shin, J., and B. Berkowitz, 1994: Prediction of ice shapes and their effect on airfoil drag. J. Aircr., 31, 263-270, https://doi.org/ $10.2514 / 3.46483$.

Starr, K. M., and T. Wang, 1989: The development of a present weather sensor for Automated Surface Observing Systems. Preprints, Third Int. Conf. on Aviation Weather Systems, Anaheim, CA, Amer. Meteor. Soc., 112-116.

Tattelman, P., 1980: Field tests of a surface ice accretion measurement system. Air Force Geophysics Laboratory Rep. AFGL-TR-81-0007, 22 pp.

- 1982: An objective method of measuring surface ice accretion. J. Appl. Meteor., 21, 599-612, https://doi.org/10.1175/ 1520-0450(1982)021<0599:AOMFMS > 2.0.CO;2.

Tobin, D. M., M. R. Kumjian, and A. W. Black, 2019: Characteristics of recent vehicle-related fatalities during active precipitation in the United States. Wea. Climate Soc., 11, 935-952, https:// doi.org/10.1175/WCAS-D-18-0110.1.

Tran, P., M. T. Brahimi, I. Paraschivoiu, A. Pueyo, and F. Tezok, 1995: Ice accretion on aircraft wings with thermodynamic effects. J. Aircr., 32, 444-446, https://doi.org/ 10.2514/3.46737.

Wade, C. G., 2003: A multisensor approach to detecting drizzle on ASOS. J. Atmos. Oceanic Technol., 20, 820-832, https://doi.org/ 10.1175/1520-0426(2003)020<0820:AMATDD>2.0.CO;2. 